\title{
Collagen type I regulates B-catenin tyrosine phosphorylation and nuclear translocation to promote migration and proliferation of gastric carcinoma cells
}

\author{
AIQING LI ${ }^{1}$, TIANHUA ZHOU ${ }^{2}$, LEI GUO ${ }^{2}$ and JIANMIN SI ${ }^{1}$ \\ ${ }^{1}$ Gastroenterology laboratory, Clinic Medical Research Institution, Sir Run Run Shaw Hospital, \\ Zhejiang University School of Medicine, Hangzhou 310016; ${ }^{2}$ The Center for Diseases Modeling, \\ Zhejiang University School of Medicine, Hangzhou 310058, P.R. China
}

Received November 9, 2009; Accepted January 11, 2010

DOI: 10.3892/or_00000757

\begin{abstract}
The interaction between tumor cells and the stroma environment has crucial effects on tumor cell invasive behavior. As a major component of the stroma, collagen plays a key role on cellular adhesion and epithelial-mesenchymal transition (EMT). Recently, we found that collagen type I is significantly up-regulated in gastric cancer tissues compared with their adjacent non-neoplastic tissues. However, whether collagen type I contributes to gastric cancer invasion and metastasis is not clear. Herein we show that, collagen type I induces cell scattering and cytoskeleton rearrangement, prompts cell migration and proliferation, which indicates that collagen type I is involved in promoting gastric cancer invasion and metastasis. Collagen type I is able to reduce cellcell adhesion and enhance migration by inducing disassembly of the E-cadherin/catenin complex in gastric carcinoma cells, which is related to tyrosine phosphorylation of $B$-catenin. Tyrosine phosphorylation of $\beta$-catenin dissociates it from E-cadherin and actin cytoskeleton and facilitates its entry into the nucleus, where $\beta$-catenin acts as a transcriptional activator inducing genes involved in cell proliferation. In conclusion, collagen type I contributes to invasion and metastasis by regulating 3 -catenin tyrosine phosphorylation and nuclear translocation to promote migration and proliferation of gastric carcinoma cells.
\end{abstract}

\section{Introduction}

The intensive communication between stroma environment and tumor cells regulates tumor cells growth, differentiation,

Correspondence to: Dr Jianmin Si, Gastroenterology laboratory, Clinic Medical Research Institution, Sir Run Run Shaw Hospital, Zhejiang University School of Medicine, Hangzhou 310016 , P.R. China

E-mail: jianmin_si@zju.edu.cn

Key words: collagen type I, cellular adhesion, epithelialmesenchymal transition, metastasis, gastric cancer and migration (1). Recent work starts to address the importance of the microenvironment in supporting invasive growth and the possible molecular mechanisms by which stroma environment contributes to tumorigenesis (2). Collagen, as a large family of triple helical proteins, is the major constituent of the tumor stroma environment. The collagen superfamily now includes more than 20 collagen types. Collagen type I and type III, as main components of extracellular matrix (ECM), play vital roles on the disruption of cellular adhesion and epithelialmesenchymal transition (EMT) (3-6), which influence tissue integrity and allow tumor cells to disseminate from the primary tumor, subsequently result in invasion and metastasis.

The invasion and metastasis are largely responsible for poor prognosis of gastric cancer. Recently, we investigated the expression of several collagen genes in gastric cancer tissues. We found that the expression levels of the two genes of collagen type I (COL1A1 and COL1A2) were significantly up-regulated in gastric cancer tissues compared with their adjacent non-neoplastic tissues (7). A better understanding of the role of stroma environment in the progression of gastric cancer will allow the development of new diagnostic and therapeutic strategies in cancer treatment. Therefore, we investigated the effects of collagen type I on gastric carcinoma cell behavior including cell morphology, cytoskeleton organization, migration and proliferation.

Malignant transformation is often characterized by major changes in the cytoskeleton organization, reducing adhesion, and aberrant adhesion signaling. The first step of invasion and metastasis involves the dysfunction of adherens junctions and the induction of migration, which resembles EMT (8-10). Cellular adhesion mediated by the E-cadherin/catenin complex represents the major cell-cell adhesion molecules in epithelial cells. It is pivotal for the dynamic regulation of the assembly of the E-cadherin/catenin complex as well as its association with the cytoskeleton to maintain strong cell-cell adhesion and differentiation epithelial tissues (11). The dysfunction of the E-cadherin/catenin complex occurs in an early stage of carcinogenesis, playing a crucial role in disruption of tissue architecture and loss of differentiation in early gastric cancer (12). B-catenin is first identified as the key component of cell-cell adhesion junctions (13). It binds to E-cadherin and, by associating with $\alpha$-catenin, provides a stable connection 
between the cytoskeleton and cell-cell junctions. Alteration in tyrosine phosphorylation of B-catenin is the most important molecular mechanism which regulates the disruption of the E-cadherin/catenin complex (14-16).

In pancreatic cancer, the alteration in tyrosine phosphorylation of $\beta$-catenin to reduce cell-cell adhesion and the activation of $\beta$-catenin/T-cell factor (TCF) signaling play a crucial role in tumor invasion and metastasis. Collagen type I initiates disassembly of the E-cadherin/catenin complex in pancreatic carcinoma cells, which is caused by tyrosine phosphorylation of the adhesion complex protein $\beta$-catenin. Tyrosine phosphorylation of $\beta$-catenin dissociates it from E-cadherin and actin cytoskeleton and facilitats its entry into the nucleus, where $\beta$-catenin actes as a transcriptional activator inducing genes involved in the initiation of cell proliferation (3). The aim of this study was to address whether these signaling pathways are involved in gastric carcinoma cell scattering, cytoskeleton reorganization, migration and proliferation induced by collagen type I.

\section{Materials and methods}

Cell culture. Three gastric carcinoma cell lines originated from various differentiation stages, BGC-823 (poorly differentiated, Type Culture Collection of Chinese Academy of Sciences, TCCCAS), SGC-7901 (moderately differentiated, TCCCAS) and NCI-N87 (well differentiated, American Type Culture Collection, ATCC) were maintained in RPMI-1640 medium supplemented with $10 \%$ fetal bovine serum (FBS). Cells ( $\left.1 \times 10^{6}\right)$ were cultured on cell culture dishes (Corning, USA) coated with collagen type I (BD, rat tail, $5 \mu \mathrm{g} / \mathrm{cm}^{2}$ ) or uncoated dishes (Control). Cells were cultured until they formed a confluent monolayer for at least $24 \mathrm{~h}$.

Reagents. Monoclonal anti-E-cadherin antibody (610181) was purchased from BD Biosciences (Lexington, KY). Monoclonal anti-ß-actin antibody (A-5441) was obtained from Sigma (St. Louis, MO). Polyclonal antibodies against E-cadherin (4065), focal adhesion kinase (FAK, 3285), B-catenin (9562), phosphatase and tensin homolog (PTEN, 9552) and anti-rabbit IgG, HRP-linked antibody (7074) were purchased from Cell Signaling (Beverly, MA). Antibody antiphospho-FAK Tyr ${ }^{397}$ (3283), anti-phospho-PTEN Ser ${ }^{380}$ (9554) were also obtained from Cell Signaling. An HRP-coupled phosphotyrosine-specific antibody (610011) was purchased from BD Biosciences. Goat anti-rabbit IgG $(\mathrm{H}+\mathrm{L})$ Alexa Fluor488 (GAR4881) and goat anti-mouse IgG (H+L) Alexa Fluor488 (GAM4881) were purchased from MultiSciences Biotech Co., Ltd. Rhodamine-conjugated phalloidin was from Invitrogen Life Technology (Carlsbad, CA).

Immunofluorescence. Cells were cultured on collagen type Icoated dishes or uncoated control dishes. From 24 h to 5 days of confluence, cells were fixed in $3.7 \%$ paraformaldehyde in PBS (pH 7.4) for 10 min, permeabilized in $0.1 \%$ Triton X-100 in PBS for $10 \mathrm{~min}$, blocked with $1 \%$ bovine serum albumin (BSA)/PBS for $1 \mathrm{~h}$, and then incubated at room temperature for $2 \mathrm{~h}$ with different antibody (against E-cadherin, rhodamineconjugated phalloidin) at 1:100 in blocking solution. E-cadherin immunoreactive proteins were visualized with
Alexa 488-coupled anti-mouse antibody. Nuclei were counterstained with DAPI. Images were acquired with an Olympus BX51 microscope.

Cell migration assay. Cells were trypsinized and resuspended in RPMI-1640 containing 5\% FBS at a density of $5 \times 10^{5}$ cells/ $\mathrm{ml}$. Collagen type I $(500 \mu \mathrm{g} / \mathrm{ml})$ was coated on the out-side membrane facing lower chamber of Millicell hanging cell culture inserts $(8.0 \mu \mathrm{m}$ pore size PET membrane, Millipore, USA). Cell suspension (100 $\mu \mathrm{l})$ was added into the upper chamber of a transwell consisted of inserts outside membranes coated with collagen type I or not. RPMI-1640 (600 $\mu 1)$ containing $20 \%$ FBS was placed in the lower chamber. After a 4-h incubation at $37^{\circ} \mathrm{C}$, cells that remained in the upper chamber were removed carefully by cotton swab and the membrane was cut off by an operating knife. The side facing lower chamber was stained with DAPI $(1 \mu \mathrm{g} / \mathrm{ml})$ and attached cells were counted under an immunofluorescence microscope. The experiment was performed 3 times.

Western blotting and immunoprecipitation. Confluent grown cells were lysed with RIPA buffer [1\% Triton X-100, 0.5\% sodium deoxycholate, $0.1 \% \mathrm{SDS}, 150 \mathrm{mM} \mathrm{NaCl}, 50 \mathrm{mM}$ Tris/ $\mathrm{HCl}$ ( $\mathrm{pH}$ 7.2), $10 \mathrm{mM}$ EDTA] or for coimmunoprecipitation analyses with immunoprecipitation buffer $[10 \mathrm{mM}$ Tris/HCl (pH 7.4), 150 mM NaCl, $1 \%$ Triton X-100, 1\% Nonidet P-40]. Both buffers contained $50 \mu \mathrm{M}$ leupeptin, $1 \mathrm{mM}$ sodium orthovanadate, $1 \mathrm{mM}$ sodium fluoride and $1 \mathrm{mM}$ PMSF (phenylmethylsulphonyl fluoride) to inhibit proteases. Homogenized lysates $(1,000-2,000 \mu \mathrm{g})$ were immunoprecipitated with $5 \mu 1$ of the indicated antibody and protein $\mathrm{A}+\mathrm{G}$-agarose (Beyotime Institute of Biotechnology). The immunoprecipitates were analyzed by SDS-PAGE and incubated with the indicated antibody for $2 \mathrm{~h}$. Nuclear fractions were prepared using Cell Protein extract kit (Beyotime Institute of Biotechnology) following the manufacturer's instructions.

Triton X-100-fractionated cell lysates were prepared as described by Hinck with minor modifications (17). Cells rinsed by ice-cold phosphate-buffered saline (PBS) were incubated with $400 \mu 1$ of Triton X-100 buffer per 100-mm dish $(0.5 \%$ Triton X-100, $0.3 \mathrm{M}$ sucrose, $100 \mathrm{mM} \mathrm{NaCl}, 3 \mathrm{mM}$ $\mathrm{MgCl}_{2}, 10 \mu \mathrm{g} / \mathrm{ml}$ leupeptin, $1 \mathrm{mM}$ PMSF for $10 \mathrm{~min}$ on a rocking platform at $4^{\circ} \mathrm{C}$. The cells were harvested with a rubber policeman, $30 \mu 1$ was removed and homogenized. The remaining lysate was cleared with $10,000 \mathrm{x} \mathrm{g}$ for $10 \mathrm{~min}$ at $4^{\circ} \mathrm{C}$. The supernatant containing the Triton X-100-soluble fraction was collected and the remaining pellet was resuspended in $400 \mu \mathrm{l}$ SDS buffer [1\% SDS, 2 mM EDTA, $20 \mathrm{mM}$ Tris (pH 7.5), $1 \mathrm{mM}$ PMSF, $1 \mu \mathrm{g} / \mathrm{ml}$ DNase I]. After centrifugation at $10,000 \mathrm{x}$ for $10 \mathrm{~min}$ at $4^{\circ} \mathrm{C}$, the supernatant was collected which contains a Triton X-100-insoluble fraction. Equal volumes of both fractions corresponding to $30 \mu \mathrm{g}$ of the Triton X-100-insoluble fraction were analyzed by Western blotting. Detection was carried out using the ECL kit (Pierce Chemical Co., Rockford, IL) and the blots were developed using a Fujifilm LAS-4000 imaging system.

Cell proliferation assay. Cell proliferation was assessed by using the MTT (methyl thiazolyl tetrazolium) assay. Cells $\left(4 \times 10^{3}\right)$ were cultured in 96-well plates coated collagen type I 


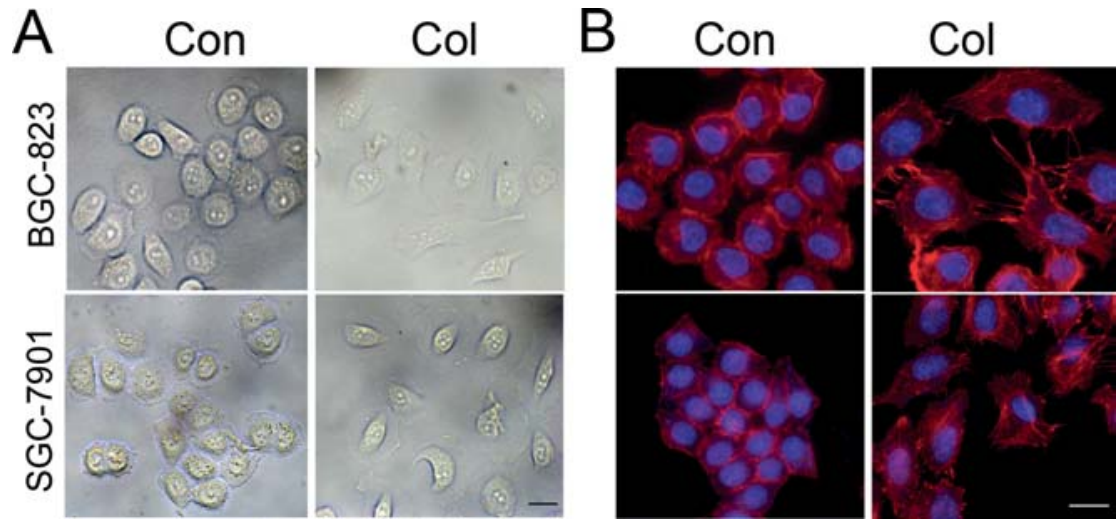

Figure 1. Morphological changes of gastric carcinoma cells in response to collagen type I. BGC-823 and SGC-7901 cells cultured on collagen type I-coated dishes (Col) or uncoated control dishes (Con) for $24 \mathrm{~h}$ were subjected to Phase contrast (A) and immunofluorescence analyses (B). Rhodamine-conjugated phalloidin (red) was used to detect F-actin fibers, and DAPI (blue) was used to visualize the nuclei. Bars, $20 \mu \mathrm{m}$.

or not. After 24, 48 and $72 \mathrm{~h}$ of incubation, $200 \mu \mathrm{l}$ of $5 \mathrm{mg} / \mu \mathrm{l}$ MTT solution (Sigma-Aldrich, St. Louis, MO) in PBS was added to each well for $4 \mathrm{~h}$. Absorbance of each well was measured on a microplate reader at a wavelength of $490 \mathrm{~nm}$. Five duplicate wells were set up for each group, and experiment was repeated 3 times.

Quantitative real-time RT-PCR. Total RNA was extracted using the TRIzol reagent (Invitrogen Co., Ltd.) following the manufacturer's instructions. Total RNA was reversely transcribed into cDNA using PrimeScript ${ }^{\mathrm{TM}} \mathrm{RT}$ reagents kit (Takara, Japan) at $37^{\circ} \mathrm{C}$ for $15 \mathrm{~min}$ and at $85^{\circ} \mathrm{C}$ for $5 \mathrm{sec}$. PCR reactions using SYBR ${ }^{\circledR}$ Premix Ex Taq $^{\mathrm{TM}}$ kit were proceeded by $95^{\circ} \mathrm{C}$ for $30 \mathrm{sec}$, followed by 40 cycles at $95^{\circ} \mathrm{C}$ for $15 \mathrm{sec}$ and $58^{\circ} \mathrm{C}$ for $15 \mathrm{sec} \mathrm{s}$ with 7500 Real-time PCR system (Applied Biosystems, USA). At the end of the PCR cycles, melting curve analyses were performed as well as electrophoresis of the products on $2 \%$ agarose gels in order to validate the specific generation of the expected PCR product. Each sample was run in triplicate for analysis. The housekeeping gene $B$-actin served as an internal control. The expression of each gene was normalized to $\beta$-actin and presented as a relative expression ratio $(\Delta c t)$. Fold changes were determined using the equation $2^{-\Delta \Delta C t}$ relative to matched reference sample. Cyclin D1: sense, 5'-ACGGCCGAGAAG CTGTGCAT-3'; antisense, 5'-TTCCAATCCGCCCTCCA TGG-3'; ß-actin: sense, 5'-GTGGCCGAGGACTTTGATTG-3'; antisense, 5'-AGTGGGGTGGCTTTTAGGATG-3' .

Statistics. Data are expressed as mean \pm standard error (SE). Student's t-test was applied to analyze the difference between groups. All statistical calculations were performed by the SPSS 13.0 for Windows (SPSS Inc., Chicago, IL, USA). P<0.05 was considered statistically significant.

\section{Results}

Collagen type I induces cell morphological changes. Gastric carcinoma cell lines BGC-823 and SGC-7901 showed apparent changes in cell morphology after $24 \mathrm{~h}$ collagen type I stimulation (Fig. 1A). Cells cultured on collagen type I exhibited elongated or enlarged shape with a higher cytoplasm/ nucleus ratio as compared with the cells directly cultured on dishes. They were resting on the collagen type I substrate with spreading of pseudopodia and a loss of cell-cell contacts typically observed in mesenchymal cells. Cells directly cultured on dishes showed smaller and more round shape.

Collagen type I induces cell cytoskeleton reorganization. Since E-cadherin/catenin complex mediated cellular adhesion and actin cytoskeleton arrangement contributed substantially to epithelial cell morphology, we further examined the impact of collagen type I on the assembly of the E-cadherin/catenin complex and actin cytoskeleton reorganization. BGC-823 and SGC-7901 cells cultured on collagen type I showed profound changes in cytoskeleton organization after $24 \mathrm{~h}$ of stimulation. Immunofluorescence microscopy demonstrated that cells grown on collagen type I were scattered with a loss of cell-cell contacts but more lamellipodia and filopodia, characteristic of great motility. In addition, prominent and elongated microfilament bundles accumulated into dense stress fibers in BGC-823 and SGC-7901 cells cultured on collagen type I (Fig. 1B). On the contrary, no obvious filopodia and only a diffuse microfilament pattern were present in BGC-823 and SGC-7901 cells directly cultured on dishes.

Collagen type I enhances cell migration. To analyze whether cell cytoskeleton reorganization induced by collagen type I is involved in the migratory behavior of gastric carcinoma cells, we performed transwell migration assays with collagen type Icoated or uncoated outer-side porous membranes. As shown in Fig. 2, BGC-823 and SGC-7901 cells showed an eight-fold increase in cell migration towards the collagen type I-coated outer-side porous membranes compared with uncoated ones. NCI-N87 cells showed a 12 -fold increase in cell migration although the number of cells, which migrated through the pores, was less than that of the other two cell lines. These results indicated collagen type I induced cell migration in a similar manner as chemotactic factors, corresponding to cell morphology change and cytoskeleton reorganization.

Collagen type I disrupts functional E-cadherin/catenin adhesion complex. Three gastric carcinoma cell lines, NCI-N87, 

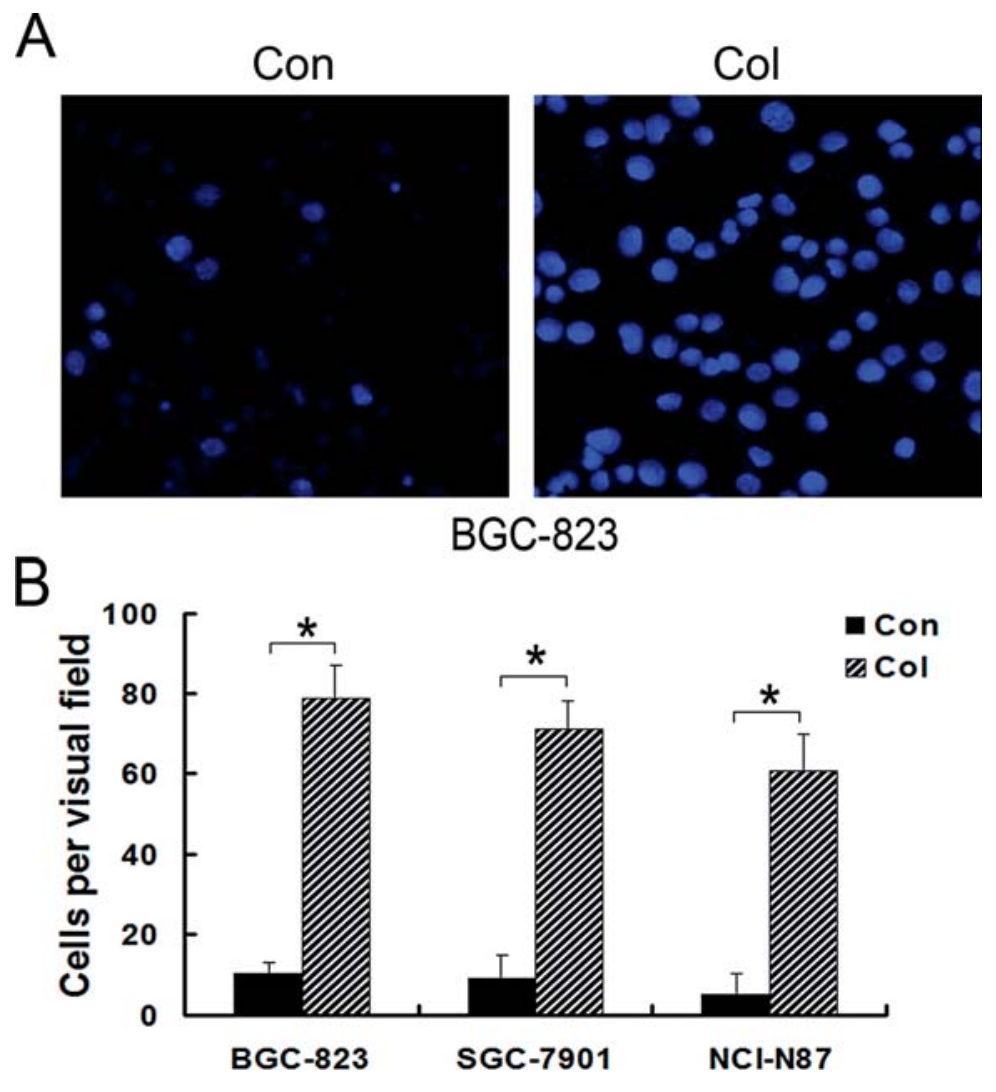

Figure 2. Collagen type I enhances gastric carcinoma cell migration. Gastric carcinoma cells were loaded into transwell inserts with outer-side membrane collagen type I-coated (Col) or not (Con). (A) BGC-823 cells that had migrated through the pores after $4 \mathrm{~h}$ of incubation were stained with DAPI. Bars, $20 \mu \mathrm{m}$. (B) The number of these cells were estimated by counting 5 independent visual fields. Columns, mean of representative experiments; Bars, \pm SE; ${ }^{*} \mathrm{P}<0.01$.

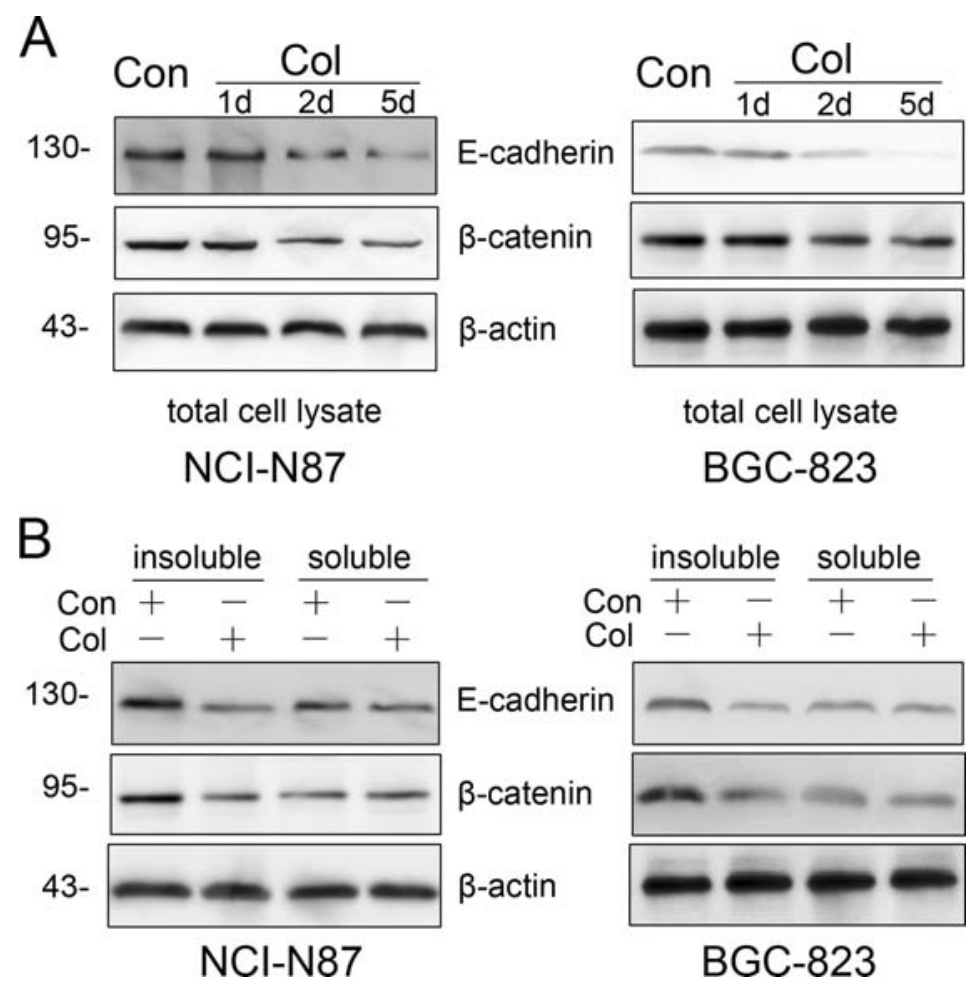

Figure 3. Collagen type I initiates disassembly of the E-cadherin/catenin adhesion complex. Gastric carcinoma cells were cultured on collagen type I-coated dishes or uncoated control dishes for different times. (A) The total lysate of cells was subjected to Western analysis with anti-E-cadherin and B-catenin antibodies. (B) Triton-soluble and -insoluble protein fractions isolated from cells treated with collagen type I or not were also analyzed by immunoblotting with the indicated antibodies. B-actin was used for loading control. One representative blot out of three independent experiments is shown in each part. 

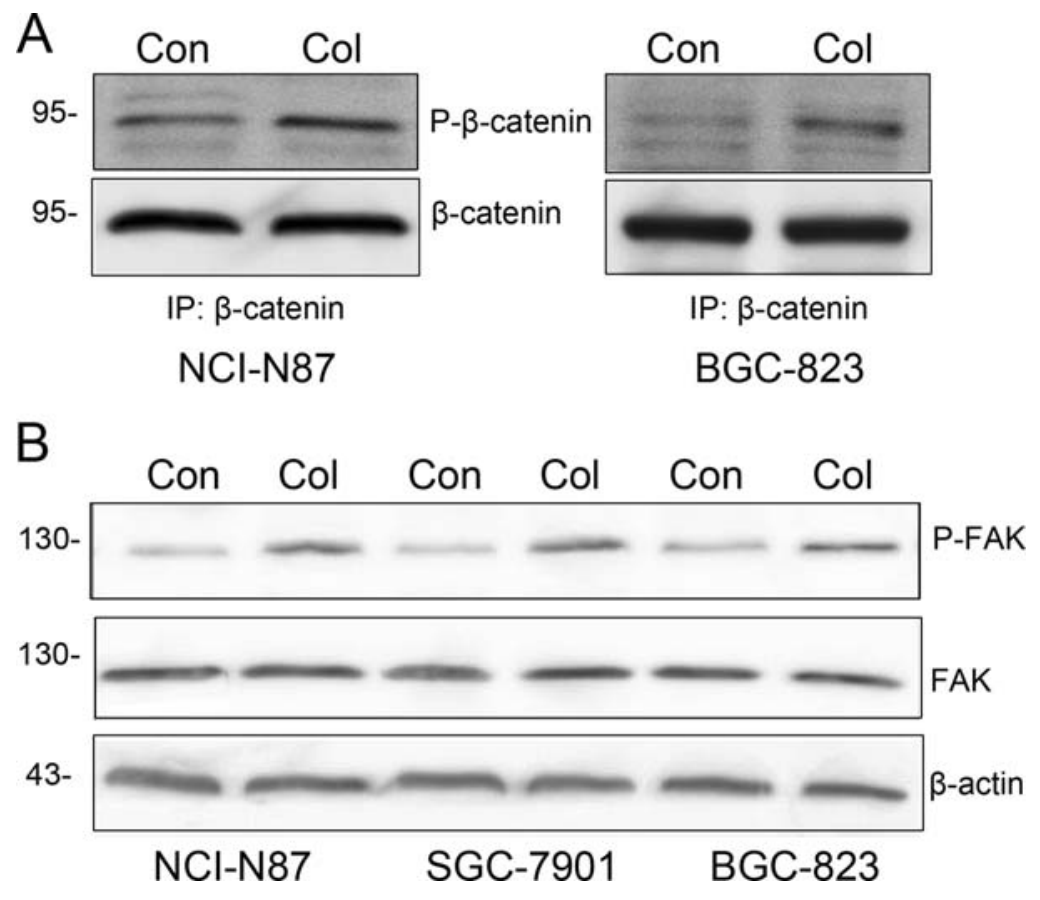

Figure 4. Collagen type I induces tyrosine phosphorylation of ß-catenin and FAK. Gastric carcinoma cells were cultured on collagen type I-coated dishes or uncoated control dishes for 2 days. (A) Total $\beta$-catenin immunoprecipitated from cell lysates was subjected to Western blotting with anti- $\beta$-catenin antibody and HRP-coupled phosphotyrosine-specific antibody. (B) Total FAK and tyrosine-phosphorylated FAK in cell lysates were examined by immunoblotting analysis with the indicated antibodies. Representative blots were from three independent experiments.

SGC-7901 and BGC-823 cells, directly cultured on dishes showed typical E-cadherin staining at membranes localised at the site of cell-cell contacts. By contrast, cells grown on the collagen type I for at least 3 days showed a loss or reduction of E-cadherin in membranes of contacting cells (data not shown). Total protein lysates of NCI-N87, SGC-7901 and BGC-823 cells cultured on coated collagen type I or uncoated dishes were analyzed regarding protein levels of E-cadherin and $\beta$-catenin. As shown in Fig. 3A, E-cadherin protein concentration in NCI-N87 and BGC-823 cells were decreased after 2 days of collagen type I treatment, and the total amount continued to reduce and reached maximum after 5-7 days. The protein concentration of B-catenin in NCI-N87 and BGC823 cells were also reduced after 2 days of collagen type I stimulation, but did not change upon collagen type I stimulation after 2 days. SGC-7901 cells exhibited similar changes in E-cadherin and $\beta$-catenin protein levels.

Next, in order to determine the amount of E-cadherin/ B-catenin complex associated with the cytoskeleton, we analysed the E-cadherin/ß-catenin distribution in Triton X-100 soluble and insoluble fractions in three cell lines, which were grown on collagen type I-coated or uncoated dishes for 2 days. As illustrated in Fig. 3B, the amounts of E-cadherin and B-catenin were reduced in Triton-insoluble fractions after collagen type I stimulation, indicating that the E-cadherin/ catenin adhesion complex associated with the actin cytoskeleton were reduced. Whereas, in the Triton-soluble fractions, the amount of E-cadherin remained constant in NCI-N87 and BGC-823 cells after collagen type I stimulation; the amount of $\beta$-catenin was slightly reduced in BGC-823 cells or remained constant in NCI-N87 cells after 2 days of collagen type I treatment. These results suggested that collagen type I treatment causes disruption of the E-cadherin/catenin adhesion complex and dissociation from the actin cytoskeleton in gastric carcinoma cells, subsequently resulting in a decrease in cellcell adhesion.

Collagen type I induces tyrosine phosphorylation of $\beta$-catenin. Tyrosine phosphorylation of $\beta$-catenin is important for regulation of E-cadherin/catenin complex assembly $(14,15)$. We studied tyrosine phosphorylation of $B$-catenin in gastric carcinama cells grown on collagen type I. To analyze the phosphorylation level of $\beta$-catenin, we chose a collagen incubation time of 2 days for immunoprecipitation analyses. Tyrosine phosphorylation was detected using a phosphotyrosine specific antibody. As shown in Fig. 4A, the phosphorylation levels of B-catenin were increased in NCI-N87 cells and BGC-823 cells cultured on collagen type I compared with that directly cultured on dishes. Comparable results were obtained using SGC-7901 cells (data not shown).

Collagen type I induces activation of FAK. Activation of the cellular tyrosine kinase FAK correlated with the phosphorylation of $\beta$-catenin (3). To study the molecular mechanism resulting in an enhanced phosphorylation of $\beta$-catenin in gastric carcinoma cells, we analyzed the role of the tyrosine kinase FAK. Although the total amount of cellular FAK after collagen type I treatment remained unchanged, a slightly increased phosphorylation of FAK at $\mathrm{Tyr}^{397}$ was found in lysates of collagen type I stimulated NCI-N87 cells (Fig. 4B), which is a major autophosphorylation site in FAK and an indicator of activation of the FAK kinase. Similar results were obtained from experiments with BGC-823 and SGC-7901 cells. 


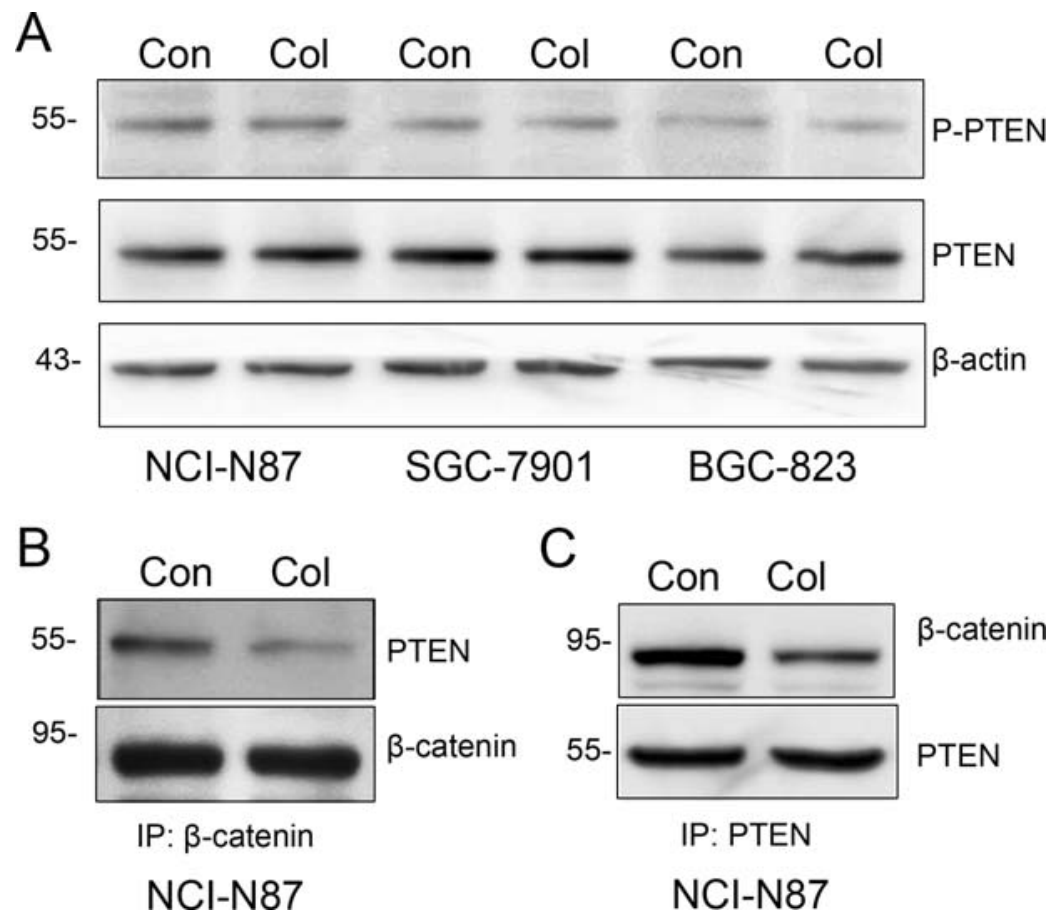

Figure 5. Collagen type I induces the dissociation of endogenous PTEN and B-catenin in vivo. Gastric carcinoma cells were cultured on collagen type I-coated dishes or uncoated control dishes for 2 days. These cells were subjected to immunoblotting with the antibodies against either total PTEN or phosphorylated PTEN (A). The PTEN coimmunoprecipitated by anti-ß-catenin antibody (B) and the $\beta$-catenin copurified with PTEN (C) were examined by Western analysis with the indicated antibodies.

Collagen type I induces the dissociation of endogenous PTEN and $\beta$-catenin. The phosphatase PTEN has been described to be involved in stabilizing cell-cell adhesion (18). Coimmunoprecipitation experiment demonstrates PTEN can directly dephosphorylate $\beta$-catenin in TGF- $\beta 1$-stimulated pancreatic carcinoma cells $(4,19)$. To analyze if PTEN was involved in the regulation of E-cadherin/catenin complex disassembly in gastric carcinoma cells, we examined the amount of PTEN and its phosphorylation. The total amount of PTEN, and its inhibitory phosphorylation at $\mathrm{Ser}^{380}$ in the lysates of NCIN87 did not change in response to collagen type I (Fig. 5A). Similarly, no significant differences were observed in the lysates of BGC-823 and SGC-7901 cells upon collagen treatment. We further immunoprecipitated PTEN and B-catenin from cell lysates and examined the amount of PTEN precipitated with B-catenin. As documented in Fig. 5B and C, collagen treatment of NCI-N87 cells resulted in a significant reduction of PTEN co-immunoprecipitating to B-catenin. Comparable results were obtained in SGC-7901 and BGC823 cells (data not shown). Together, these results indicated that PTEN associated with $\beta$-catenin were markedly reduced in gastric carcinoma cells upon collagen treatment, and PTEN might be involved in the regulation of phosphorylation of ß-catenin.

Collagen type I promotes proliferation of gastric carcinoma cells. We investigated the effect of collagen type I on the proliferation of cells derived from gastric cancer. The proliferation rates of cells cultured on collagen type I-coated or uncoated dishes were evaluated by MTT assays. Except changes of cell morphology and motility, the proliferation rate of BGC-823 cells and SGC-7901 cells grown on collagen type I were significantly increased compared with the controls on dishes (Fig. 6A). Similar results were obtained in NCI-N87 cells with a clear increase in the proliferation rate.

Proliferation induced by collagen type I is related with $\beta$-catenin nuclear translocation. $\beta$-catenin is not only involved in the regulation of cell-cell adhesion as a component of the E-cadherin/catenin complex in the cell membrane, but also mediated the proliferating signal through the $\beta$-catenin-LEF/ TCF pathway (20). B-catenin acts as a transcription cofactor with lymphoid enhancer factor/T cell factor (LEF/TCF) in the $\beta$-catenin-LEF/TCF pathway. We compared the amounts of $\beta$-catenin in nuclear fraction and total cell lysate grown on collagen type I or directly dishes. As shown in Fig. 6B, NCIN87 cells stimulated with collagen exhibited a significant increase of B-catenin in the nuclear fraction compared with the cells grown on dishes. We also observed a clear increase of $\beta$-catenin in the nuclear fractions of SGC-7901 and BGC823 cells which were cultured on collagen type I (data not shown). These data indicated that collagen type I promoted translocation of $ß$-catenin into the nucleus in gastric carcinoma cells.

To determine whether this translocation of $\beta$-catenin induced by collagen type I influences physiological LEF/TCF target genes, we analyzed the amount of cyclin D1 mRNA by quantitative real-time RT-PCR. In line with the data obtained from pancreatic carcinoma cells, the mRNA concentrations of cyclin D1 were increased in three gastric carcinoma cells cultured on collagen type I compared with the controls on dishes (Fig. 6C). Interestingly, although NCI-N87 cells with 

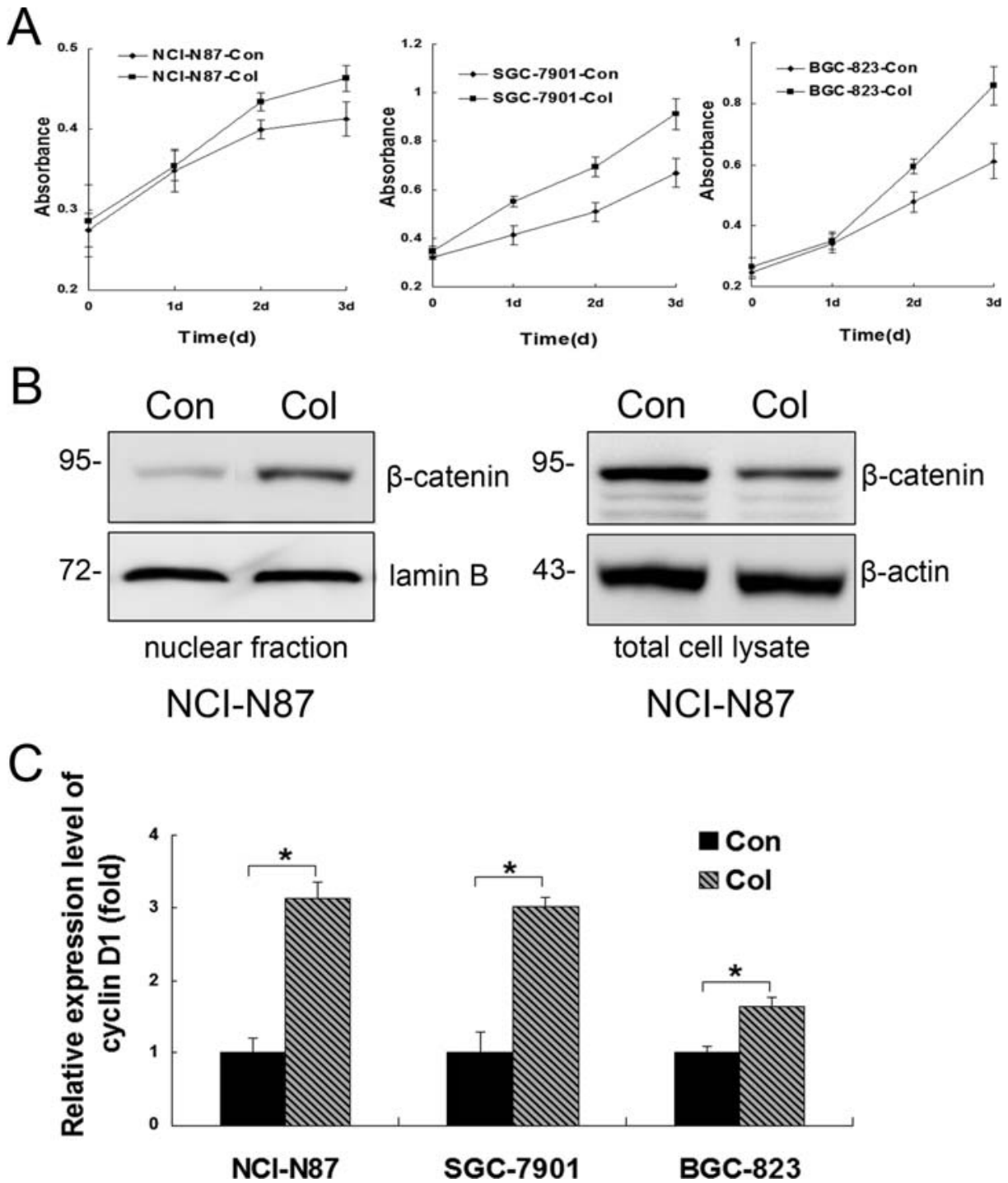

Figure 6. Collagen type I promotes gastric cancer cell proliferation. Gastric carcinoma cells were cultured on collagen type I-coated dishes or uncoated control dishes for 3 days. (A) The effects of collagen type I on the proliferation of gastric carcinoma cells were measured by MTT assay. The same number of cells were seeded and cultured for 3 days. At each time point, the cells were assayed for proliferation. (B) The amount of $\beta$-catenin from nuclear extracts and total cell lysates of NCI-N87 cells were analyzed by Western blotting. (C) Real-time quantitative RT-PCR analysis of the relative expression level of cyclin D1 mRNA in gastric carcinoma cells. Three independent experiments were performed. Bars, $\pm \mathrm{SE}$; ${ }^{*} \mathrm{P}<0.05$.

collagen treatment showed a significant increase of ß-catenin in nuclear translocation and increase of cyclin D1 mRNA expression, NCI-N87 cells exhibited a smaller increase in the proliferation rate upon collagen stimulation than the SGC7901 and BGC-823 cells.

\section{Discussion}

Induction of EMT represents a primary step during progression from a localized tumor to an invasive carcinoma. In this process, cancer cells not only change their adhesive repertoire, but also gain migratory and invasive properties that involves a dramatic cytoskeleton remodeling and the concomitant formation of membrane protrusions (21). In this study, we found that collagen type I induced gastric carcinoma cell transition from epithelial cell to mesenchymal-like cell accompanying with cytoskeleton remodeling and migration enhancing. During the transition process, cancer cells possessed powerful proliferation and various migratory organelles required for invasive growth, such as lamellipodia and filopodia. At molecular level, cell-cell adhesion molecule E-cadherin and E-cadherin/catenin adhesion complex were reduced or lost while ß-catenin/T-cell factor (TCF) proliferation signaling was activated.

To become motile in processes such as wound healing and tumor invasion, epithelial cells undergo EMT with a concomitant disruption of E-cadherin/catenin adhesion. Much work has been done to explore the molecular mechanisms involved in the regulation of E-cadherin complex assembly. These mechanisms are highly diverse, including altered expression of genes coding for adhesion proteins and altered interaction or modulation of adhesion proteins (22-25). The impact of $\beta$-catenin phosphorylation on regulation of the E-cadherin/catenin adhesion complex assembly has been demonstrated in different studies. We found that collagen type I induced the tyrosine phos- 
phorylation of $\beta$-catenin, then the phosphorylated $\beta$-catenin dissociated itself from E-cadherin and actin cytoskeleton. Furthermore, increased tyrosine phosphorylation of B-catenin was accompanied by phosphorylation of FAK. This is in line with findings reported by Irby et al, that FAK plays a role in the assembly of the E-cadherin complex in cultured colon cancer cells $(26,27)$. Accumulated data show that the ECM, which are produced by many tumors, support tumor invasion by activating integrin signaling, whereas FAK is one of the important molecules (28). FAK participates in the disruption of E-cadherin/catenin complex by phosphorylation of $\beta$-catenin $(3,4,15)$.

The phosphatase PTEN has been described to interact with the E-cadherin complex and mediated dephosphorylation and localization of B-catenin $(19,29)$. In pancreatic carcinoma cells, the membranous localization of PTEN is reduced after stimulation by collagen type I. The concentration of E-cadherin/ catenin associated PTEN is also reduced while its inhibitory phosphorylation is increased in cells cultured on collagen type I (30). Whether PTEN is also involved in the regulation of E-cadherin/catenin complex disassembly in gastric carcinoma cells is not clear. Our study revealed that PTEN and its inhibitory phosphorylation remained unchanged after collagen stimulation, while the capacity that PTEN associated with B-catenin was down-regulated, which suggested that the amount of PTEN involved in dephosphorylation of $\beta$-catenin is decreased. This led to increased phosphorylation of $\beta$-catenin and disruption of E-cadherin complex after collagen treatment. These molecular changes basically elucidated cell scattering, cytoskeleton remodeling and subsequent migration change.

Besides the effects on cell scattering, cytoskeleton remodeling and migration change, collagen type I prompted proliferation of gastric carcinoma cells. In this study, we showed a collagen type I-induced nuclear translocation of B-catenin and an increased cellular proliferation rate in gastric carcinoma cells. EMT of the tumor cells is associated with a nuclear accumulation of the transcriptional activator $\beta$-catenin (2). The nuclear $\beta$-catenin localization results in up-regulating of $\beta$-catenin/LEF/TCF transcriptional activator and concomitant expression of many targeted tumorigenic gene products, such as cyclin D1 (31). There is evidence that the up-regulation of cyclin D1 mRNA level correlates with cell proliferation (3). Activated $\beta$-catenin/LEF/TCF complex has been identified to increase not only the proliferation but also the migration of epithelial cells (32). The expression level of cyclin D1 was up-regulated in gastric carcinoma cells after collagen stimulation. The up-regulation was not only involved in the $B$-catenin/LEF/TCF signaling pathway, but also in other related pathways, since NCI-N87 cells exhibited a smaller increase in proliferation rate after collagen stimulation compared to the other two cell lines despite the fact that its expression level of cyclin D1 was higher.

In conclusion, our data demonstrated that collagen type I induced cell scattering and cytoskeleton rearrangement, and enhanced migration and proliferation of gastric carcinoma cells. E-cadherin/catenin-mediated adhesion pathway and ß-catenin/T-cell factor (TCF) pathway were involved in these processes. Collagen type I contributes to invasion and metastasis by regulating $\beta$-catenin tyrosine phosphorylation and nuclear translocation to promote migration and proliferation of gastric carcinoma cells. These findings would help to develop a new therapy strategy against gastric cancer invasive and metastatic events in future.

\section{Acknowledgements}

This study was supported by National High Technology Research and Development Program of China (863 Program) (No. 20060102Z4055). We thank Dr Xiaotong Hu (Key Laboratory of Biotherapy of Zhejiang Province, Sir Run Run Shaw Hospital, Zhejiang University School of Medicine) for technical assistance and helpful discussion.

\section{Reference}

1. De Wever O and Mareel M: Role of tissue stroma in cancer cell invasion. J Pathol 200: 429-447, 2003.

2. Brabletz T, Hlubek F, Spaderna S, et al: Invasion and metastasis in colorectal cancer: epithelial-mesenchymal transition, mesenchymal-epithelial transition, stem cells and beta-catenin. Cells Tissues Organs 179: 56-65, 2005.

3. Koenig A, Mueller C, Hasel C, Adler G and Menke A: Collagen type I induces disruption of E-cadherin-mediated cell-cell contacts and promotes proliferation of pancreatic carcinoma cells. Cancer Res 66: 4662-4671, 2006.

4. Imamichi Y and Menke A: Signaling pathways involved in collagen-induced disruption of the E-cadherin complex during epithelial-mesenchymal transition. Cells Tissues Organs 185: 180-190, 2007

5. Shintani Y, Maeda M, Chaika N, Johnson KR and Wheelock MJ: Collagen I promotes epithelial-to-mesenchymal transition in lung cancer cells via transforming growth factor-beta signaling. Am J Respir Cell Mol Biol 38: 95-104, 2008.

6. Kirkland SC: Type I collagen inhibits differentiation and promotes a stem cell-like phenotype in human colorectal carcinoma cells. Br J Cancer 101: 320-326, 2009.

7. Zhao Y, Zhou T, Li A, et al: A potential role of collagens expression in distinguishing between premalignant and malignant lesions in stomach. Anat Rec (Hoboken) 292: 692-700, 2009.

8. Thiery JP: Epithelial-mesenchymal transitions in tumour progression. Nat Rev Cancer 2: 442-454, 2002.

9. Huber MA, Kraut N and Beug H: Molecular requirements for epithelial-mesenchymal transition during tumor progression. Curr Opin Cell Biol 17: 548-558, 2005.

10. Larue L and Bellacosa A: Epithelial-mesenchymal transition in development and cancer: role of phosphatidylinositol 3' kinase/ AKT pathways. Oncogene 24: 7443-7454, 2005.

11. Cowin P, Rowlands TM and Hatsell SJ: Cadherins and catenins in breast cancer. Curr Opin Cell Biol 17: 499-508, 2005.

12. Joo YE, Rew JS, Choi SK, Bom HS, Park CS and Kim SJ: Expression of E-cadherin and catenins in early gastric cancer. J Clin Gastroenterol 35: 35-42, 2002.

13. Kemler R: From cadherins to catenins: cytoplasmic protein interactions and regulation of cell adhesion. Trends Genet 9: 317-321, 1993.

14. Nelson WJ and Nusse R: Convergence of Wnt, beta-catenin, and cadherin pathways. Science 303: 1483-1487, 2004.

15. Lilien $\mathrm{J}$ and Balsamo $\mathrm{J}$ : The regulation of cadherin-mediated adhesion by tyrosine phosphorylation/dephosphorylation of beta-catenin. Curr Opin Cell Biol 17: 459-465, 2005.

16. Ostman A, Hellberg C and Bohmer FD: Protein-tyrosine phosphatases and cancer. Nat Rev Cancer 6: 307-320, 2006.

17. Hinck L, Nathke IS, Papkoff J and Nelson WJ: Dynamics of cadherin/catenin complex formation: novel protein interactions and pathways of complex assembly. J Cell Biol 125: 1327-1340, 1994.

18. Kotelevets L, van Hengel J, Bruyneel E, Mareel M, van Roy F and Chastre E: The lipid phosphatase activity of PTEN is critical for stabilizing intercellular junctions and reverting invasiveness. J Cell Biol 155: 1129-1135, 2001.

19. Vogelmann R, Nguyen-Tat MD, Giehl K, Adler G, Wedlich D and Menke A: TGFbeta-induced downregulation of E-cadherinbased cell-cell adhesion depends on PI3-kinase and PTEN. J Cell Sci 118: 4901-4912, 2005. 
20. Behrens J, von Kries JP, Kuhl M, et al: Functional interaction of beta-catenin with the transcription factor LEF-1. Nature 382: 638-642, 1996

21. Yilmaz M and Christofori G: EMT, the cytoskeleton, and cancer cell invasion. Cancer Metastasis Rev 28: 15-33, 2009.

22. Troyanovsky SM: Mechanism of cell-cell adhesion complex assembly. Curr Opin Cell Biol 11: 561-566, 1999.

23. D'Souza-Schorey C: Disassembling adherens junctions: breaking up is hard to do. Trends Cell Biol 15: 19-26, 2005.

24. Reynolds $\mathrm{AB}$ and Roczniak-Ferguson $\mathrm{A}$ : Emerging roles for p120-catenin in cell adhesion and cancer. Oncogene 23: 7947-7956, 2004

25. Menke A, Philippi C, Vogelmann R, et al: Down-regulation of E-cadherin gene expression by collagen type I and type III in pancreatic cancer cell lines. Cancer Res 61: 3508-3517, 2001.

26. Irby RB and Yeatman TJ: Increased Src activity disrupts cadherin/catenin-mediated homotypic adhesion in human colon cancer and transformed rodent cells. Cancer Res 62: 2669-2674, 2002.
27. Avizienyte E and Frame MC: Src and FAK signalling controls adhesion fate and the epithelial-to-mesenchymal transition. Curr Opin Cell Biol 17: 542-547, 2005.

28. Giehl K and Menke A: Microenvironmental regulation of E-cadherin-mediated adherens junctions. Front Biosci 13: 3975-3985, 2008.

29. Persad S, Troussard AA, McPhee TR, Mulholland DJ and Dedhar S: Tumor suppressor PTEN inhibits nuclear accumulation of beta-catenin and T cell/lymphoid enhancer factor 1-mediated transcriptional activation. J Cell Biol 153: 1161-1174, 2001.

30. Raftopoulou M, Etienne-Manneville S, Self A, Nicholls S and Hall A: Regulation of cell migration by the $\mathrm{C} 2$ domain of the tumor suppressor PTEN. Science 303: 1179-1181, 2004.

31. Brabletz T, Jung A and Kirchner T: Beta-catenin and the morphogenesis of colorectal cancer. Virchows Arch 441: 1-11, 2002.

32. van Es JH, Barker $\mathrm{N}$ and Clevers $\mathrm{H}$ : You Wnt some, you lose some: oncogenes in the Wnt signaling pathway. Curr Opin Genet Dev 13: 28-33, 2003. 